\title{
Congruences on distributive pseudocomplemented lattices
}

\section{Willlam H. Cornish}

Let $B_{0} \subset B_{1} \subset B_{2} \subset \ldots \subset B_{n} \subset \ldots \subset B_{\omega}$ be all the non-trivial varieties of distributive pseudocomplemented lattices $(L ; \vee, \wedge, *, 0,1)$ considered as algebras of type $(2,2,1,0,0)$. A subset $J$ of such an algebra $L$ is a congruence-kernel if and only if it is a lattice-ideal and $x^{* *} \in J$ for each $x \in J$. The smallest congruence having $J$ as its kernel is $\Theta(J)$, where $a \equiv b(\Theta(J)),(a, b \in L)$ if and only if $a \wedge c^{*}=b \wedge c^{*}$ for some $c \in J$. For given $0 \leq n \leq \omega$, let $\Sigma_{n}(J)$ be the smallest congruence having $J$ as its kernel and such that the associated quotient algebra is in $B_{n}$. Of course, $\Sigma_{\omega}(J)=\theta(J)$ and the main result of this paper shows that for $1 \leq n<\omega$,

$$
\begin{aligned}
\Sigma_{n}(J)=n\left\{\theta\left(P_{1} \cap P_{2} \cap \ldots \cap P_{n}\right): J \subseteq\right. & P_{1}, P_{2}, \ldots, P_{n} \subset L \\
& \text { are minimal prime ideals }\} .
\end{aligned}
$$

It is also shown that $\Sigma_{\omega}(J)$ is the smallest congruence on the lattice $(L ; \vee, \wedge, 0, I)$ having $J$ as its kernel for each congruence kernel $J$ if and only if $L \in B_{1}$. On the other hand, for any congruence kernel $J, \Sigma_{0}(J)$ is always the largest congruence on the lattice $(L ; v, \wedge, 0,1)$ having $J$ as its kernel and so $\Sigma_{0}(J)$ is the unique congruence with $J$ as its kernel and such that quotient algebra is boolean. Received 10 October 1972. 


\section{Congruence-kerne]s}

A congruence $\theta$ on a distributive pseudocomplemented lattice $(L ; \vee, \wedge, *, 0,1)$ is a congruence of the lattice $(L ; \vee, \wedge, 0,1)$ which also has the substitution property for the *-operation. At times it will be convenient to refer to such a congruence as a ${ }^{*}$-congruence. For such a congruence $\theta, \operatorname{ker}(\theta)=\{x \in L: x \equiv 0(\theta)\}$ and $\operatorname{coker}(\theta)=\{x \in L: x \equiv I(\theta)\}$ are called, respectively, the kermel and cokernel of $\theta$. A subset $J$ of $L$ is called a congruence-kermel (respectively congruence-cokernel) if $J=\operatorname{ker}(\theta)$ (respectively $J=\operatorname{coker}(\theta)$ ) for some *-congruence $\theta$ on $L$.

The purpose of this section is to characterize the congruence-kernels of a given algebra $(L ; \vee, \wedge, *, 0,1)$ and also to describe the smallest and largest congruences on $L$ which have a given congruence-kernel as their common kernel. The necessary prerequisite information on the variety of all distributive pseudocomplemented lattices can be obtained from Chapter 3 of Grätzer's recent book [4]. We begin with some preliminary results on distributive lattices which will be useful both in this section and in the later sections.

Let $J$ be an ideal in a distributive lattice $L$. Then a prime ideal $P$ in $L$ is called a minimal prime ideal belonging to $J$ if $P$ is a minimal member of the set of all prime ideals in $L$ which contain $J$. If $L$ has a smallest member, 0 , then a minimal prime ideal of $L$ is simply a minimal prime ideal belonging to the zero-ideal (0). The following lemma summarizes some useful information which appears in the literature.

LEMMA 1.1. The following results hold in a distributive lattice $L$.

(i) A prime ideal $P$ containing an ideal $J$ is a minimal prime ideal belonging to $J$ if and only if, for each $x \in P$, there exists $y \in L \backslash P$ such that $x \wedge y \in J$.

(ii) Suppose that $0 \in L$. and that $Q$ is a prime ideal in $L$. Then, $O(Q)=\{x \in L: x \wedge y=0$ for some $y \in L \backslash Q\}$ is the intersection of all the minimal prime ideals of $L$ which are contained in $Q$.

(iii) If $L$ is pseudocomplemented then a prime ideal $P$ is a minimal prime ideal if and only if $x^{* *} \in P$ for each $x \in P$; and the set of all minimal prime ideals of $L$ endowed with the 
hull-kernel topology, is a compact Hausdorff space.

Proof. (i) follows from [7, Lemma 3.1] and the distributivity of $L$.

(ii) is established in [1, Proposition 2.2].

The first part of (iii) follows from (i), see also [4, Lemma 5 , p. 169], while the second part is a consequence of [9, Proposition 3.2].

If $J$ is an ideal in a distributive lattice $L$ then $\Theta_{\text {Lat }}(J)$ and $R(J)$ will be used to respectively denote the smallest congruence and the largest congruence on $L$ possessing $J$ as a congruence class. It is well-known that $x \equiv y\left(\Theta_{\text {Lat }}(J)\right),(x, y \in L)$, if and only if

$x \vee a=y \vee a$ for some $a \in J$, while $x \equiv y(R(J)),(x, y \in L)$, if and only if, for any $b \in L, x \wedge b \in J$ if and only if $y \wedge b \in J$. For detailed information on $\Theta_{\text {Lat }}(J)$ and $R(J)$ we refer respectiveiy to [4] and [10]. In [10, Proposition 4.1] it is shown that if $1 \in[$ then $\{x \in L: x \equiv I(R(J))\}$ is the filter $D(J)$ where $D(J)=\{a \in L: J: a=J\}$ and $J: a$ is the ideal $\{b \in L: a \wedge b \in J\}$. When $0 \in L, R$ will be used to denote $R((0))$ and $D$ will be used to denote $D((0])$. If $L$ is pseudocomplemented then $x \equiv y(R)$, $(x, y \in L)$, if and only if $x^{* *}=y^{* *}$ and $D=\left\{x \in L: x^{* *}=1\right\}$, the filter of dense elements.

LEMMA 1.2. Let $h(J)$ denote the set of all minimal prime ideals belonging to a given ideal $J$ in a distributive lattice $L$. Then

(i) $R(J)=n\{R(P): P \in h(J)\}$, and

(ii) $\Theta_{\text {Lat }}(J)=n\left\{\Theta_{\text {Lat }}(P): P \in h(J)\right\}$.

Proof. (i) Suppose that $x, y \in L$ and $x \equiv y(R(J))$. If $x \in P \in h(J)$ then, according to Lemma $1 . l(i), x \wedge z \in J$ for some $z \in L \backslash P$. Then $y \wedge z \in J$ and so $y \in P$. By symmetry, we conclude that $x \in P$ if and only if $y \in P$, that is, $x \equiv y(R(P))$. Thus, $R(J) \subseteq n\{R(P): P \in h(J)\}$. But $J=\Pi\{P: P \in h(J)\}$ and so $J$ is a congruence class of $L$ modulo $\cap\{R(P): P \in h(J)\}$. Hence $n\{R(P): P \in h(J)\} \subseteq R(J)$. Part (i) is now proved.

(ii) For any $P \in h(J)$, it is clear that $\Theta_{\text {Lat }}(J) \subseteq \Theta_{\text {Lat }}(P)$. 
Suppose that $x, y \in L$ and $x \neq 1$ y $\left(\Theta_{\text {Lat }}(J)\right)$ and consider $F=\{a \in L: x \vee a=y \vee a\}$. Then $F$ is non-empty since $x \vee y \in F$, and $F$ is a filter. By the hypothesis on $x$ and $y$, it follows that $J$ and $F$ are disjoint. Using the Birkhoff-Stone Theorem and the dual form of Zorn's Lemma, it follows that there exists $Q \in h(J)$ such that $Q$ and $F$ are disjoint. Hence, $x \neq y(\Theta(Q))$. The equality asserted in $(i i)$ is now readily established.

If $F$ is a filter in an arbitrary lattice $L$ then the relation $\Psi(F)$ defined by: $x \equiv y(\Psi(F))(x, y \in L)$ if and only if $x \wedge f=y \wedge f$ for some $f \in F$, is always an equivalence relation. When $L$ is a distributive lattice, $\Psi(F)$ is the smallest congruence having $F$ as a congruence class. Special cases of the following lemma are well-known, for example, [5, Lemma 7, p. 36] and [6, Theorem 2]; we include a simple direct proof.

LEMMA 1.3. For any two filters $F_{1}$ and $F_{2}$ in a lattice $L$, the equivalence relations $\Psi\left(F_{1}\right)$ and $\Psi\left(F_{2}\right)$ are permutable.

Proof. Suppose that $x, y \in L$ and that $x \equiv y\left(\Psi\left(F_{1}\right) \circ \Psi\left(F_{2}\right)\right)$. Then, $x \wedge f_{1}=z \wedge f_{1}$ and $z \wedge f_{2}=y \wedge f_{2}$ for some $z \in L, f_{1} \in F_{1}$ and $f_{2} \in F_{2}$. Thus, $x \wedge f_{1} \wedge f_{2}=y \wedge f_{1} \wedge f_{2} \cdot$ Put $w=\left(x \wedge f_{2}\right) \vee\left(y \wedge f_{1}\right)$. Then $x=x \vee\left(x \wedge f_{1} \wedge f_{2}\right)=x \vee\left(y \wedge f_{1} \wedge f_{2}\right)$ $\equiv\left(x \wedge f_{2}\right) \vee\left(y \wedge f_{1}\right)=w\left(\Psi\left(F_{2}\right)\right)$, and $w=\left(x \wedge f_{2}\right) \vee\left(y \wedge f_{1}\right) \equiv\left(x \wedge f_{1} \wedge f_{2}\right) \vee y=y\left(\Psi\left(F_{1}\right)\right)$. That is, $x \equiv y\left(\Psi\left(F_{2}\right) \circ \Psi\left(F_{1}\right)\right)$. The lemma now follows from symmetry.

The next result describes congruence-cokernels in a distributive pseudocomplemented lattice; it will be useful throughout the entire paper.

PROPOSITION 1.4. Let $(L ; v, \wedge, *, 0,1)$ be a distributive pseudocomplemented lattice. A subset $F$ of $L$ is a congruence-cokernel if and only if $F$ is a filter in $L$. Moreover, the smallest *-congruence having a given filter $F$ as its cokernel is $\Psi(F)$. 
Proof. It is sufficient to prove that, for a given filter $F, \Psi(F)$ has the substitution property for the *-operation. Thus suppose that $x, y \in L$ and $x \equiv y(\Psi(F))$, that is, $x \wedge f=y \wedge f$ for some $f \in F$. Then $x^{* *} \wedge f^{* *}=(x \wedge f)^{* *}=(y \wedge f)^{* *}=y^{* *} \wedge f^{* *}$ so that $x^{*} \underline{v} f^{*}=y^{*} \underline{v} f^{*}$ in the skeleton $(S(L) ; \underline{v}, \wedge, *, 0, l)$ of $L$. Then $x^{*} \wedge f^{* *}=y^{*} \wedge f^{* *}$, since $S(L)$ is a boolean algebra, and hence $x^{*} \wedge f=y^{*} \wedge f$.

If $J$ is an ideal in a distributive psuedocomplemented lattice $L$, let $J_{*}=\left\{x \in L: x \geq a^{*}\right.$ for some $\left.a \in J\right\}$. It is easy to see that $J_{*}$ is a filter. We now come to our first characterization of congruencekernels.

THEOREM 1.5. Let $J$ be a non-empty subset of a distributive pseudocomplemented lattice $(L ; \vee, \wedge, *, 0,1)$. Then the following conditions are equivalent:

(i) $J$ is a congruence-kernel,

(ii) $J$ is an ideal of the lattice $L$ and $x^{* *} \in J$ for each $x \in J$,

(iii) $J$ is an ideal of the lattice $L$ and each minimal prime ideal belonging to $J$ is a minimal prime ideal,

(iv) $J$ is an intersection of minimal prime ideals of the lattice $L$.

Moreover, if $J$ satisfies any of the equivalent conditions (i)-(iv) then the smallest congmence on $(L ; V, \Lambda, *, 0,1)$ having $J$ as its kernel is $\Theta(J)$, where $x \equiv y(\Theta(J))(x, y \in L)$ if and only if $x \wedge a^{*}=y \wedge a^{*}$ for some $a \in J$. Thus, $\Theta(J)=\Psi\left(J_{*}\right)$.

Proof. $(i) \Rightarrow(i i)$. Suppose that $J=\operatorname{ker}(\theta)$ for some *-congruence $\theta$. Then $J$ is certainly a lattice-ideal and, for $x \in J, x \equiv 0(\theta)$, so that $x^{* *} \equiv 0^{* *}=0(\theta)$, and hence (ii) holds for $J$.

$(i i) \Rightarrow(i)$. From (ii) and Proposition 1.4, $\Psi\left(J_{*}\right)$ is a *-congruence. Because $x^{* *} \in J$ for $x \in J, \operatorname{ker}\left(\Psi\left(J_{*}\right)\right)=J$. If $\Phi$ is a *-congruence with $\operatorname{ker}(\Phi)=J$ then $x \wedge a^{*}=y \wedge a^{*}$ for $a \in J$ implies $x \equiv y(\Phi)$ since $a^{*} \equiv 0^{*}=1(\Phi)$. Thus, $(i i) \Rightarrow(i)$, and we have also described the smallest *-congruence having $J$ as its kernel. 
The implication (iv) $\Rightarrow$ (ii) follows from Lemma 1.1 (iii) so that it remains to establish that $(i i) \Rightarrow$ (iii) and (iii) $\Rightarrow$ (iv).

$(i i) \Rightarrow(i i i)$. Let $J$ satisfy $(i i)$ and suppose that $P$ is a minimal prime ideal belonging to $J$. Let $x \in P$. By Lemma 1.1 (i), $x \wedge z \in J$ for some $z \in L \backslash P$. Then $x^{* *} \wedge z^{* *}=(x \wedge z)^{* *} \in J$ and so $x^{* *} \in P$, since $P$ is prime and $z \leq z^{* *}$. Then, by Lemma 1.1 ( $\left.i i i\right), P$ is a minimal prime ideal.

$(i i i) \Rightarrow(i v)$. Each ideal in a distributive lattice is the intersection of all the minimal prime ideals belonging to it. Hence (iv) follows from (iii).

THEOREM 1.6. Let $J$ be a given congmence-kernel in a distributive pseudocomplemented lattice $(L ; v, \wedge, *, 0,1)$. The following conditions relating to an equivalence relation $\Phi$ on $L$ are equivalent:

(i) $\Phi$ is the Zargest *-congruence such that $J=\operatorname{ker} \Phi$,

(ii) $\Phi=R(J)$,

(iii) for cony $x, y \in L, x \equiv y(\Phi)$ if and only if $x^{* *} \wedge a^{*}=y^{* *} \wedge a^{*}$ for some $a \in J$,

(iv) $\Phi=\Theta(J) \vee R$ in the lattice of (*-)congmences,

(v) for any $x, y \in L, x \equiv y(\Phi)$ if and only if $x \wedge\left(b \vee b^{*}\right) \wedge a^{*}=y \wedge\left(b \vee b^{*}\right) \wedge a^{*}$ for some $b \in L$ and $a \in J$,

(vi) $\Phi$ is the smallest *-congruence with $J=\operatorname{ker} \Phi$ and such that $L / \Phi$ is a boolean algebra,

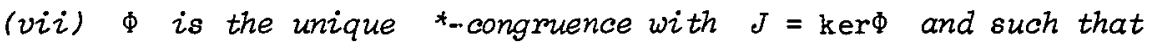
$L / \Phi$ is a boolean algebra.

Proof. $(i) \Leftrightarrow(i i)$. By Theorem 1.5, each minimal prime ideal belonging to $J$ is a minimal prime ideal. Hence, by Lemma $1.1, R(P)$ is a *-congruence for each $P \in h(J)$ and so Lemma 1.2 implies that $R(J)$ is

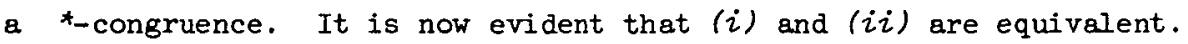

(iii) $\Leftrightarrow(v)$. Because $\left(b \vee b^{*}\right)^{* *}=1$ for each $b \in L$, (iii) follows from $(v)$. On the other hand suppose that $x^{* *} \wedge a^{*}=y^{* *} \wedge a^{*}$ for some $a \in J$. Since $x \vee x^{*}$ and $y \vee y^{*}$ are dense, 
$\left(x \vee x^{*}\right) \wedge\left(y \vee y^{*}\right)=b \vee b^{*}$ for some $b \in L$ and then

$x \wedge\left(b \vee b^{*}\right) \wedge a^{*}=\left(x^{* *} \wedge\left(x \vee x^{*}\right)\right) \wedge\left(b \vee b^{*}\right) \wedge a^{*}$

$$
=x^{* *} \wedge\left(b \vee b^{*}\right) \wedge a^{*}=y^{* *} \wedge\left(b \vee b^{*}\right) \wedge a^{*}=y \wedge\left(b \vee b^{*}\right) \wedge a^{*} \text {. }
$$

Thus, (iii) and (v) are equivalent.

(iv) $\Leftrightarrow(v)$. Because $L$ is pseudocomplemented, $R=\Psi(D)$, (cf. [10, Proposition 5.4]). Thus, from Theorem 1.5,

$$
\Theta(J) \vee R=\Psi\left(J_{*}\right) \vee \Psi(D)=\Psi\left(J_{*} \vee D\right),
$$

where

$$
J_{*} \vee D=\left\{x \in L: x=b \wedge d, b \in J_{*}, d \in D\right\}
$$

is the join of $J_{*}$ and $D$ in the lattice of filters on $L$. Hence (iv) and $(v)$ are equivalent.

(ii) $\Longleftrightarrow(i i i)$. Because of the equivalence of (iii), (iv), and (v) it is clear that the relation $\Phi$ of (iii) is a *-congruence with $\operatorname{ker} \Phi=J$ and hence $\Phi \subseteq R(J)$. It remains to establish the reverse inequality.

Let $P$ be a minimal prime ideal and suppose that $x \equiv y(R(P))$ for given $x, y \in L$. Then $x, y \in P$ or $x, y \in L \backslash P$. In the first case $x \vee y \in P$ and $x^{* *} \wedge(x \vee y)^{*}=x^{* *} \wedge x^{*} \wedge y^{*}=0=y^{* *} \wedge(x \vee y)^{*}$, while in the second case $x \wedge y \in L \backslash P$ so that $(x \wedge y)^{*} \in P$ and $x^{* *} \wedge\left((x \wedge y)^{*}\right)^{*}=x^{* *} \wedge(x \wedge y)^{* *}=x^{* *} \wedge y^{* *}=y^{* *} \wedge\left((x \wedge y)^{*}\right)^{*}$. Thus, for any minimal prime ideal $P, x \equiv y(R(P))(x, y \in L)$ if and only if $x^{* *} \wedge p^{*}=y^{* *} \wedge p^{*}$ for some $p \in P$.

Now suppose $x, y \in L$ and $x \equiv y(R(J))$. Let $h(J)=\left\{P_{\lambda}: \lambda \in \Lambda\right\}$ be the set of all minimal prime ideals containing $J$. By Lemma 1.2 and Theorem 1.5, $x \equiv y\left(R\left(P_{\lambda}\right)\right)$ for each $\lambda \in \Lambda$. It follows from the preceding paragraph that, for each $\lambda \in \Lambda$, there exists $p_{\lambda} \in P_{\lambda}$ such that $x^{* *} \wedge p_{\lambda}^{*}=y^{* *} \wedge p_{\lambda}^{*}$. Hence, for each $P_{\lambda} \in h(J)$, $P_{\lambda} \in g\left(p_{\lambda}^{*}\right)=\left\{Q: Q\right.$ is minimal prime ideal and $\left.p_{\lambda}^{*} \mid Q\right\}$. That is, $\left\{g\left(p_{\lambda}^{*}\right): \lambda \in \Lambda\right\}$ is an open cover of the closed subset $h(J)$ of the space of minimal prime ideals. Because of Lemma 1.1 (iii), $h(J)$ is compact and so there exist $\lambda_{1}, \lambda_{2}, \ldots, \lambda_{n} \in \Lambda$ such that 


$$
h(J) \subseteq g\left(p_{\lambda_{1}}^{*}\right) \cup g\left(p_{\lambda_{2}}^{*}\right) \cup \ldots \cup g\left(p_{\lambda_{n}^{*}}\right)=g\left(p_{\lambda_{1}^{*}} \vee p_{\lambda_{2}}^{*} \vee \ldots \vee p_{\lambda_{n}}^{*}\right)
$$

Hence

$$
\begin{array}{r}
p_{\lambda_{1}^{*}}^{*} \wedge \ldots \wedge p_{\lambda_{n}^{* *}}^{*}=\left(p_{\lambda_{1}}^{*} \vee \ldots \vee p_{\lambda_{n}}^{*}\right)^{*} \in \cap\left\{Q: Q \in g\left(p_{\lambda_{1}^{*}}^{*} \vee \ldots \vee p_{\lambda_{n}}^{*}\right)\right\} \\
\subseteq n\{Q: Q \in h(J)\}=J,
\end{array}
$$

by Theorem 1.5. Now $x^{* *} \wedge p_{\lambda_{i}^{*}}^{*}=y^{* *} \wedge p_{\lambda_{i}}^{*}$ for $i=1, \ldots, n$ and so

$$
x^{* *} \wedge\left(p_{\lambda_{1}}^{*} \underline{v} \cdots \underline{v} p_{\lambda_{n}}^{*}\right)=y^{* *} \wedge\left(p_{\lambda_{1}}^{*} \underline{v} \cdots \underline{v} p_{\lambda_{n}}^{*}\right)
$$

in $S(L)$. Hence

$$
x^{* *} \wedge\left(p_{\lambda_{1}^{* *}}^{*} \ldots \wedge p_{\lambda_{n}^{* *}}^{*}\right)^{*}=y^{* *} \wedge\left(p_{\lambda_{1}^{* *}}^{*} \ldots \wedge p_{\lambda_{n}^{* *}}^{*}\right.
$$

and $p_{\lambda}^{* *} \wedge \ldots \wedge p_{\lambda}^{* *} \in J$. Thus $x \equiv y(\Phi)$, where $\Phi$ is the congruence of (iii). And (ii) and (iii) are equivalent.

Thus conditions $(i)$ to $(v)$ are equivalent.

$(v) \Leftrightarrow(v i)$. If $\Phi$ is the congruence of $(v)$ then $\operatorname{ker}(\Phi)=J$ and $b \vee b^{*} \epsilon \operatorname{coker}(\Phi)$ for each $b \in L$ and so $L / \Phi$ is boolean. It is clear that $\Phi$ is the smallest *-congruence with these properties and so $(v)$ and (vi) are equivalent.

$(v i) \Leftrightarrow(v i i)$. Since $(i)$ and $(v i)$ are equivalent, (vii) is equivalent to $(v i)$.

The theorem has now been established.

The preceding theorem shows that the congruence $R(J)$ has a very simple description when $J$ is a congruence-kernel. In fact many of the properties of the congruence $R(J)$ which are implied by that theorem are characteristic of congruence-kernels.

THEOREM 1.7. The following conditions on an ideal $J$ of a distributive pseudocomplemented lattice $L$ are equivalent:

(i) $J$ is a congruence-kernel,

(ii) $R(J)$ is a *-congmence, 
(iii) for any $x, y \in L, x \equiv y(R(J))$ if and only if $x^{* *} \wedge a^{*}=y^{* *} \wedge a^{*}$ for some $a \in J$,

(iv) for any $x, y \in L, x \equiv y(R(J))$ if and only if $x \wedge\left(b \vee b^{*}\right) \wedge a^{*}=y \wedge\left(b \vee b^{*}\right) \wedge a^{*}$ for some $b \in L$ and $a \in J$,

(v) $R(J)=\Psi(D(J))$,

(vi) $D(J)=D \vee J_{*}$ in the lattice of filters,

(vii) $D \subseteq D(J)$,

(viii) for any fizter $F$, the congruences $R(J)$ and $\Psi(F)$ are permutable,

(ix) the congruences $R(J)$ and $R$ are permutable,

(x) $R \subseteq R(J)$

Proof. The equivalence of conditions (i), (ii), (iii) and (iv) is an easy consequence of Theorems 1.6 and 1.5 .

$(i v) \Rightarrow(v)$. Condition (iv) implies that $R(J)=\Psi(\operatorname{coker} R(J))$, and, as has been previously mentioned, $\operatorname{coker}(R(J))=D(J)$.

$(v) \Rightarrow(i i)$. This follows from Proposition 1.4 .

$(v) \Rightarrow(v i)$ and $(v i) \Rightarrow(v i i)$ are obvious.

(vii) $\Rightarrow(i)$. Suppose that $D \subseteq D(J)$ and let $x \in J$. Then $x \vee x^{*} \in D(J)$ and so $x^{* *} \wedge\left(x \vee x^{*}\right)=x$ implies $x^{* *} \in J: x=J$. Thus (i) holds.

$(v) \Rightarrow$ (viii) follows from Lemm 1.3 .

(viii) $\Rightarrow$ (ix) since $R=\Psi(D)$ holds in any psuedocomplemented lattice.

$(i x) \Rightarrow(i)$. Let $x \in J$. Then $0 \equiv x(R(J))$ and $x \equiv x^{* *}(R)$ so that $0 \equiv x^{* *}(R(J) \circ R)$. By $(i x), 0 \equiv z(R)$ and $z \equiv x^{* *}(R(J))$. Hence $z=0$ and so $x^{* *} \in J=\operatorname{ker} R(J)$.

(ii) $\Rightarrow(x)$ is clear and $(x) \Rightarrow(i)$ since $x \equiv x^{* *}(R)$ for any $x \in L$. The theorem is proved.

Some other consequence of Theorem 1.5 will be given in the next sections. 


\section{The congruences $\Sigma_{n}(J)$}

Because of Theorem 1.5, the congruence-kernels of a distributive pseudocomplemented lattice are precisely its $\alpha$-ideals, in the sense of [2]. The following lemma is thus an easy consequence of [2, Propositions $3.2,3.3]$; it is easy to provide a direct proof and so details are omitted.

LEMMA 2.1. The set $K(L)$ of all congruence-kermels of a distributive pseudocomplemented lattice $(L ; v, \Lambda, *, 0,1)$ is a complete distributive lattice, where the meet is set-theoretic intersection and the join of a family $\left\{J_{\lambda}: \lambda \in \Lambda\right\}$ of congruence-kernels is $\left.\underline{\bigvee} J_{\lambda}: \lambda \in \Lambda\right\}=\left\{x \in L: x \leq\left(a_{\lambda_{1}} \vee \ldots \vee a_{\lambda_{n}}\right\}^{* *}\right.$

$$
\text { for some } \left.a_{\lambda_{1}} \in J_{\lambda_{1}}, \ldots, a_{\lambda_{n}} \in J_{\lambda_{n}} \text { and } \lambda_{1}, \ldots, \lambda_{n} \in \Lambda\right\} \text {. }
$$

Moreover, the maps $J \rightarrow\left\{b \in S(L): b=x^{* *}\right.$ for some $\left.x \in J\right\}$ and $I \rightarrow\left\{x \in I: x^{* *} \in I\right\}$ are mutually inverse lattice-isomorphisms between $K(L)$ and the lattice of ideals of the skeleton $(S(L) ; \underline{v}, \wedge, *, 0,1)$.

LEMMA 2.2. Let $\Theta$ be a *-congruence on a distributive pseudocomplemented lattice $(L ; \vee, \wedge, *, 0, I)$ and let $\theta: L \rightarrow L / \theta$ be the canonical epimorphism. Then the set-function induced by $\theta$ and the inverse image map $\theta^{\star}$ are mutually inverse lattice isomorphisms between the interval $[\operatorname{ker} \Theta, L]=\{J \in K(L): \operatorname{ker} \Theta \leq J\}$ of the lattice $K(L)$ of congmence-kermels of $L$ and the lattice $K(L / \theta)$ of congruence-kernels of $L / \Theta$. Hence the map $P \rightarrow \theta(P)$ is a bijection of the set of minimal prime ideals of $L$ which contain ker $\Theta$ onto the set of minimal prime ideals of $L / \theta$.

Proof. Let $J \in K(L)$ be such that $\operatorname{ker} \Theta \subseteq J$ and suppose that $x \in \theta^{+} \theta(J)$. Hence $\theta(x)=\theta(a)$ for some $a \in J$, and so $\theta\left(x \wedge a^{*}\right)=\theta(x) \wedge \theta(a)^{*}=0$ implies that $x \wedge a^{*} \in \operatorname{ker} \Theta \subseteq J$. If $P$ is a minimal prime ideal containing $J$ it follows from Lemma 1.1 that $x \in P$. Because of Theorem 1.5, $x \in J$ and so $\theta^{\leftarrow} \theta(J)=J$.

It is now clear that the first statement of the lemma holds and from this we immediately obtain the second statement, because Theorem 1.5 and 
Lerma 2.1 imply that the minimal prime ideals of $L$ are precisely the meet-irreducible elements of $K(L)$.

REMARK. Lemma 2.2 gives an estimate of the way in which the lattice of congruence-kernels fails to determine the lattice of *-congruences. Of course, the map $\theta \rightarrow \operatorname{ker}(\theta)$ is a bijection (Iattice-isomorphism) of the lattice of *-congruences onto the lattice of congruence-kernels of $L$ if and only if $(L ; \vee, \wedge, *, 0, l)$ is precisely the skeleton $(S(L) ; \underline{v}, \wedge, *, 0,1)$ that is, if and only if $L$ is a boolean algebra. This is because $\operatorname{ker}(R)=(0)$ if and only if $x=x^{* *}$ for each $x \in L$.

Let $\left\{B_{n}: 0 \leq n \leq \omega\right\}$ be all the non-trivial varieties of distributive pseudocomplemented lattices (ef. [4, Chapter 3] and [8]). Thus $B_{0}$ is the class of boolean algebras, $B_{1}$ is the class of Stone lattices and $B_{\omega}$ is the class of all distributive pseudocomplemented lattices. We will need the following characterization of $B_{n} \quad(1 \leq n<w)$ due to lee $[8$, Theorems 4,5$]$.

LEMMA 2.3. Let $(L ; \vee, \wedge, *, 0,1)$ be a distributive pseudocomplemented lattice and let $1 \leq n<\omega$ be a positive integer. Then the following conditions are equivalent:

(i) $L \in B_{n}$,

(ii) any $x_{1}, x_{2}, \ldots, x_{n} \in L$ satisfy the identity

$$
\left(\bigwedge_{i=1}^{n} x_{i}\right)^{*} \vee \bigvee_{i=1}^{n}\left(x_{1} \wedge \ldots \wedge x_{i-1} \wedge x_{i}^{*} \wedge x_{i+1} \wedge \ldots \wedge x_{n}\right) *=1 \text {, }
$$

(iii) each prime ideal in $L$ contains at most $n$ distinct minimal prime ideals.

LEMMA 2.4. Let $(L ; \vee, \wedge, *, 0,1)$ be a distributive pseudocomplemented lattice. Then

(i) for any $n$ (not necessarily distinct) minimal prime ideals

$$
P_{1}, P_{2}, \ldots, P_{n} \text { in } L, L / \Theta\left(P_{1} \cap P_{2} \cap \ldots \cap P_{n}\right) \in B_{n},
$$

(ii) for any $1 \leq n<\omega, L \in B_{n}$ implies that 
$\cap\left\{\Theta\left(P_{1} \cap P_{2} \cap \ldots \cap P_{n}\right): P_{1}, P_{2}, \ldots, P_{n}\right.$ are $n$ minimal prime ideals $\}$

$$
=\omega_{L}
$$

(the smallest congmence on $L$ ).

Proof. (i) $L / \Theta\left(P_{1} \cap P_{2} \cap \ldots \cap P_{n}\right)$ has, at most, $n$ distinct minimal prime ideals. This follows from Lemma 2.2 since $P_{1}, P_{2}, \ldots, P_{n}$ are the only minimal prime ideals of $L$ containing $P_{1} \cap P_{2} \cap \ldots \cap P_{n}$. Thus part (i) follows from Lemma 2.3.

(ii) The dual of Lemma 1.2 (ii) implies that

$$
\cap\{\Psi(F): F \text { is a prime filter in } L\}=\omega_{L} \text {. }
$$

Recall that ideal $P$ is prime if and only if $L \backslash P$ is a prime filter. By Lemme 1.4, $\Psi(L \backslash P)$ is a *-congruence and it is clear that $\operatorname{ker}(\Psi(L \backslash P))=O(P)$ and so $\Theta(O(P)) \subseteq \Psi(L \backslash P)$. Combining this information we obtain: $\cap\{\Theta(O(P)): P$ is a prime ideal $\}=\omega_{L}$. Then, part $(i i)$ is an easy consequence of Lemma 2.3 (iii) and Lemma 1.1 (ii).

Let $J$ be a congruence-kernel in a distributive pseudocomplemented lattice $L$. For any $0 \leq n \leq \omega$, let $\Sigma_{n}(J)$ denote the smallest of all *-congruences $\theta$ on $L$ such that $\operatorname{ker}(\theta)=J$ and $L / \theta \in B_{n}$, so that $L / \Sigma_{n}(J)$ is the maximal homomorphic image of $L$ which lies in $B_{n}$. Even though $\Sigma_{\omega}(J)=\theta(J)$ and $\Sigma_{0}(J)=R(J)$ (Theorem 1.6), it will at times be convenient to use that notation $\Sigma_{\omega}(J)$ and $\Sigma_{0}(J)$. We will also use $\Sigma_{n}$ in place of $\Sigma_{n}((0))$. The existence and description of $\Sigma_{n}(J)$ is the aim of the next theorem. Granting the existence it is clear that

$$
\Theta(J)=\Sigma_{\omega}(J) \subseteq \cdots \subseteq \Sigma_{n}(J) \subseteq \Sigma_{n-1}(J) \subseteq \cdots \subseteq \Sigma_{I}(J) \subseteq \Sigma_{0}(J)=R(J) .
$$$$
\text { For } I \leq n<\omega \text {, let } D_{n} \text { be the filter of } L \text { generated by all }
$$
elements of the form

$$
\left(\bigwedge_{i=1}^{n} x_{i}\right)^{*} \vee \bigvee_{i=1}^{n}\left(x_{1} \wedge \ldots \wedge x_{i-1} \wedge x_{i}^{*} \wedge x_{i+1} \wedge \ldots \wedge x_{n}\right)^{*}
$$


where $x_{1}, \ldots, x_{n} \in L$. Thus

$$
\begin{aligned}
D_{n}=\left\{x \in L: x \geq \bigwedge_{j=1}^{m}\right. & \left(\bigwedge_{i=1}^{n} x_{i j}\right\}^{*} \\
& \left.\vee \bigwedge_{i=1}^{n}\left(x_{1 j} \wedge \ldots \wedge x_{i-1 j} \wedge x_{i j}^{*} \wedge x_{i+1 j} \wedge \ldots \wedge x_{n j}\right)^{*}\right\} \\
& \text { for some } \left.x_{i j},(i=1, \ldots, n, j=1, \ldots, m), \text { in } L\right\} .
\end{aligned}
$$

For any $x_{1}, \ldots, x_{n} \in L$, it is easy to see that

$$
\begin{aligned}
\left(x_{1} \wedge \ldots \wedge x_{n}\right) * \vee \bigvee_{i=1}^{n}\left(x_{1} \wedge \ldots \wedge x_{i-1} \wedge x_{i}^{*} \wedge \ldots \wedge x_{n}\right) * & \\
& \geq\left(x_{1} \wedge \ldots \wedge x_{n}\right) * \vee\left(x_{1} \wedge \ldots \wedge x_{n}\right) * *
\end{aligned}
$$

and so $D_{n} \supseteq D_{1} \supseteq D$ for any $1 \leq n \leq \omega$.

THEOREM 2.5. Let $1 \leq n<\omega$ be a given positive integer and let $J$ be a congmence-kernel in a distributive pseudocomplemented lattice $(L ; v, \wedge, *, 0,1)$. The following conditions relating to a *-congmence $\Phi$ are equivalent:

$$
\begin{aligned}
& \text { (i) } \Phi=\Sigma_{n}(J) \text {, } \\
& \text { (ii) for any } x, y \in L, x \equiv y(\Phi) \text { if and only if } \\
& x \wedge b \wedge a^{*}=y \wedge b \wedge a^{*} \text {, where } \\
& \left.b=\bigwedge_{j=1}^{m}\left(\bigwedge_{i=1}^{n} b_{i j}\right)^{*} \vee \bigvee_{i=1}^{n}\left(b_{1 j} \wedge \ldots \wedge b_{i-1 j} \wedge b_{i j}^{*} \wedge b_{i+1, j} \wedge \ldots \wedge b_{n j}\right)^{*}\right) \\
& \text { for some } b_{i j}(i=1, \ldots, n, j=1, \ldots, m) \text { in } L \text {, and } \\
& a \in J \text {, } \\
& \text { (iii) } \Phi=\Psi\left(D_{n} \vee J_{*}\right) \text {, } \\
& \text { (iv) } \Phi=\Sigma_{n} \vee \theta(J) \text { in the lattice of (*-)congruences, } \\
& \text { (v) } \\
& \Phi=n\left\{\theta\left(P_{1} \cap P_{2} \cap \ldots \cap P_{n}\right): P_{1}, P_{2}, \ldots, P_{n}\right. \text { are (not necessarily. } \\
& \text { distinct) minimal prime ideals in } L \text { which contain } J\} \text {. }
\end{aligned}
$$


Proof. It is clear that (ii) and (iii) are equivalent. The congruence $\Phi$ given in both is, indeed, a *-congruence (Proposition 1.4) and has $J$ as its kemel since $D_{n} \subseteq D$. As $D_{n} \subseteq \operatorname{coker}(\Phi), L / \Phi \in B_{n}$ in view of Lemma 2.3 (ii). It follows that $\Phi=\Sigma_{n}(J)$ and so $(i)$, (ii) and (iii) are equivalent.

The equivalence of ( $i i i)$ and ( $i v)$ follows from Theorem 1.5 and the equivalence of $(i)$ and ( $i i i)$ for the case $J=(0]$.

Now let $\Phi$ be the congruence given in $(v)$. The theorem will be established if we can show that $\Phi=\Sigma_{n}(J)$.

For any $n$ minimal prime ideals $P_{1}, \ldots, P_{n}$, $L / \Theta\left(P_{1} \cap \ldots \cap P_{n}\right) \in B_{n}$, by Lemma $2.4(i)$, and since $B_{n}$ is a variety, $L / \Phi \in B_{n}$, as $L / \Phi$ is a subdirect product of algebras in $B_{n}$. But $\operatorname{ker} \Phi=J \quad$ (Theorem 1.5) and so $\Sigma_{n}(J) \subseteq \Phi$ by the definition of $\Sigma_{n}(J)$. Hence it remains to show that $\Phi \subseteq \Sigma_{n}(J)$.

Let $\sigma: L \rightarrow L / \Sigma_{n}(J)$ denote the cononical epimorphism and, for congruence $\Theta \supseteq \Sigma_{n}(J)$, let $\Theta^{\prime}$ denote the congruence on $L / \Sigma_{n}(J)$ induced by $\theta$. For $\sigma(x), \sigma(y)$ in $L / \Sigma_{n}(J),(x, y \in L), x \equiv y\left(\theta^{\prime}\right)$ if and only if $x \equiv y(\theta)$; for details of this construction see [3, $\$ 1]$, Chapter 3].

Since $\Sigma_{n}(J) \subseteq \Phi, \Phi^{\prime}$ is a well-defined congruence on $L / \Sigma_{n}(J)$. Using Theorem 1.5 it follows that

$$
\begin{aligned}
\Phi^{\prime} \subseteq n\left\{\Theta \left(\sigma\left(P_{1} \cap P_{2} \cap \ldots \cap P_{n}\right):\right.\right. & P_{1}, P_{2}, \ldots, P_{n} \\
& \text { are minimal primes in } L \text { containing } J\} .
\end{aligned}
$$

As $\operatorname{ker}\left(\Sigma_{n}(J)\right)=J$, Lemma 2.2 implies that

$\Phi^{\prime} \subseteq n\left\{\theta\left(Q_{1} \cap Q_{2} \cap \ldots \cap Q_{n}\right): Q_{1}, Q_{2}, \ldots, Q_{n}\right.$

are minimal prime ideals in $\left.L / \Sigma_{n}(J)\right\}$.

Hence, by Lemma 2.4 (ii), $\Phi^{\prime}=\omega_{L / \Sigma_{n}}(J)$ and so $\Phi=\Sigma_{n}(J)$. 
Some easy consequences of the above theorem are:

COROLLARY 2.6. For any $0 \leq n<\omega, D_{n+1} \subseteq D_{n} \subseteq D$.

COROLLARY 2.7. For any $0 \leq n \leq \omega, \quad \Sigma_{0}(J)=\Sigma_{n}(J) \vee \Sigma_{0}$.

Proof. Combine Theorems 1.6 and 2.5.

COROLLARY 2.8. For any $0 \leq n, m \leq \omega$ and congruence-kemels $I$ and $J$, the congruences $\Sigma_{n}(I)$ and $\Sigma_{m}(J)$ are permutable.

Proof. Combine Lemma 1.3, Theorem 1.5, Theorem 1.6 and Theorem 2.5.

COROLLARY 2.9. For any $I \leq n<\omega$ and congmuence-kermel $J$, $L / \Theta(J) \in B_{n}$ if and only if

$$
\begin{aligned}
\Theta(J)=n\left\{\Theta\left(P_{1} \cap P_{2} \cap \ldots \cap P_{n}\right\}: P_{1}, P_{2}, \ldots, P_{n}\right. \\
\quad \text { are minimal prime ideals containing } J\} .
\end{aligned}
$$

We end this section with the following result.

THEOREM 2.10. For any fixed $0 \leq n \leq \omega$, the mop $J \mapsto \Sigma_{n}(J)$ is an injection of the lattice of congmence-kemels into the lattice of *-congruences which preserves arbitrary joins. When $n=0$, the map preserves finite meets.

Proof. It easily follows from Theorem 1.5 and Lemma 2.1 that $\theta\left(\underline{V}\left\{J_{\lambda}: \lambda \in \Lambda\right\}\right)=\bigvee\left\{\Theta\left(J_{\lambda}\right): \lambda \in \Lambda\right\}$ for any set $\left\{J_{\lambda}: \lambda \in \Lambda\right\}$ of congruence-kernels. As a consequence, Theorem 2.5 (iv) and Theorem 1.6 (iv) imply that $J \rightarrow \Sigma_{n}(J)$ preserves arbitrary joins for any $0 \leq n \leq \omega$. The last statement of the theorem follows from Theorem 1.6 (iii).

In the next section we will consider conditions on $L$ which ensure that the map $J \mapsto \Sigma_{n}(J)$ preserves finite meets even when $1 \leq n \leq \omega$.

\section{Stone lattices}

LEMMA 3.1. Let $J$ be a congmence-kernel in the distributive pseudocomplemented lattice $L$. Then $\Theta(J)=\theta_{\text {Lat }}(J)$ if and only if $J=\left\{x \in J: J \vee\left(x^{*}\right]=L\right\}$.

Proof. Suppose that $\theta(J)=\theta_{\text {Lat }}(J)$. Thus, $x \in J$ implies 
$x^{*} \equiv 1\left(\Theta_{\text {Lat }}(J)\right)$ and so $a \vee x^{*}=a \vee 1=1$ for some $a \in J$. Consequently, $\left\{x \in J: J \vee\left(x^{*}\right]=L\right\}=J$.

Conversely, suppose that $J=\left\{x \in J: J \vee\left(x^{*}\right]=L\right\}$. Suppose that $a, b \in L$ are such that $a \equiv b(\Theta(J))$. Then, by Theorem 1.5, $a \wedge x^{*}=b \wedge x^{*}$ for some $x \in J$. Using the hypothesis imposed on $J$ and working in the lattice of ideals of $L$, we obtain $(a] \vee J=((a] \vee J) \cap L=((a] \vee J) \cap\left(\left(x^{*}\right] \vee J\right)=\left((a] \cap\left(x^{*}\right]\right) \vee J$ $=\left((b] \cap\left(x^{*}\right]\right) \vee J=(b] \vee J$.

Hence $a \equiv b\left(\Theta_{\text {Lat }}(J)\right)$ and so $\theta(J)=\theta_{\text {Lat }}(J)$.

It should be noted that any ideal $J$ of the distributive pseudocomplemented lattice $L$ is necessarily a congruence-kernel if $J=\left\{x \in J: J \vee\left(x^{*}\right]=L\right\}$, and we will refer to such ideals as special congruence-kernels. It is easy to see that $L$ is a Stone lattice, that is, $L \in B_{1}$, if and only if each congruence-kernel is a special congruence-kernel. Using this observation we now come to our final result.

THEOREM 3.2. The following conditions on a distributive pseudocomplemented lattice $(L ; \vee, \wedge, *, 0,1)$ are equivalent:

(i) I is a Stone lattice,

(ii) each congmence-kermel in $L$ is a special congmence-kernel, (iii) $\theta(J)=\theta_{\text {Lat }}(J)$ for each congruence-kernel $J$,

(iv) $\Theta_{\text {Lat }}(J)$ is a *-congruence for each congruence-kerme $Z J$,

(v) $\theta(P)=\theta_{\text {Lat }}(P)$ for each minimal prime ideal $P$,

(vi) $\Theta_{\text {Lat }}(P)$ is a *-congmuence for each minimal prime ideal $P$,

(vii) each minimal prime ideal is a special congruence-kermel, (viii) $\Pi\{\theta(P): P$ is a minimal prime $i$ deal $\}=\omega_{L}$,

(ix) for any congmuence-kerme $J$ and any fizter $F, \theta_{\text {Lat }}(J)$ and $\Psi(F)$ are permutable congruences,

(x) for any congruence-kernels $I$ and $J$, $\theta(I \cap J)=\theta(I) \cap \theta(J)$, 
(xi) $L \in B_{n}$ for some $1<n<\omega$ and, given any congruence-kermels $I$ and $J, \Sigma_{n}(I) \cap \Sigma_{n}(J)=\Sigma_{n}(I \cap J)$,

(xii) for any $0 \leq n \leq \omega$, the mop $J \rightarrow \Sigma_{n}(J)$ is a zattice monomorphism of the lattice of congruence-kermels into the Zattice of *-congruences.

Proof. The equivalence of conditions (i), (ii), (iii) and (iv) follows from Lemma 3.1 as does the equivalence of conditions $(v),(v i)$ and (vii).

Condition (iii) implies $(v)$ by specializiation and $(v) \Rightarrow(v i i i)$ follows from Lemma 1.2 (ii).

Condition (viii) implies (i) by Theorem 2.5, and so the first eight conditions are equivalent.

Condition ( $i x)$ follows from ( $i v)$, by virtue of Lema 1.3 and Theorem 1.5 .

Suppose that $(i x)$ holds. Let $x \in L$ and set $J=\left(x^{* *}\right]$ and $E=[x]$. Then $J$ is a congruence-kernel, $0 \equiv x\left(\Theta_{\text {Lat }}(J)\right)$, and $x \equiv I(\Psi(F))$ so that $0 \equiv I\left(\Theta_{\text {Lat }}(J) \circ \Psi(F)\right)$. By $(i x)$, there exists $a \in L$ such that $0 \equiv a(\Psi(F))$ and $a \equiv 1\left(\Theta_{\text {Lat }}(J)\right)$. Hence $0=a \wedge x$, that is, $a \leq x^{*}$, and $a \vee x^{* *}=1$. Thus $(i x) \Rightarrow(i)$ holds and the first nine conditions are equivalent.

(iii) $\Rightarrow(x)$ is clear and $(x) \Rightarrow$ (vii) follows from Theorem 2.5 (iv), Theorem 1.6 (iv) and the distributivity of the lattice of $\left(*_{-}\right)$congruences. of course, $(x i i) \Rightarrow(x)$ and $(x) \Rightarrow(i)$, because setting $I=\left(x^{*}\right]$ and $J=\left(x^{*}\right]$ for given $x \in L$ implies that

$$
x^{*} \vee x^{* *} \equiv I(\theta(I) \cap \theta(J))=\theta(I \cap J)=\theta((0])=\omega_{L} .
$$

Since $(i)$ and (xii) are equivalent, ( $x i)$ follows from (i) and the theorem will have been proved if we show that ( $x i$ ) implies (i).

Suppose that $(x i)$ holds. Put $I=\left(x^{*}\right]$ and $J=\left(x^{* *}\right]$ for given $x \in L$. Then for $1<n<\omega$ (fixed),

$$
\Sigma_{n}(I) \cap \Sigma_{n}(J)=\Sigma_{n}(I \cap J)=\Sigma_{n}((0])=\omega_{L},
$$


as $L \in B_{n}$ (Theorem 2.5). By Theorem 2.10,

$$
\text { - } \Sigma_{n}(I) \vee \Sigma_{n}(J)=\Sigma_{n}(I \underline{v} J)=\Sigma_{n}(L) \text {, }
$$

as $x^{*} \underline{v} x^{* *}=1$, and so $\Sigma_{n}(I) \vee \Sigma_{n}(J)=I_{L}$, the largest *-congruence on $L$. Corollary 2.8 says that $\Sigma_{n}(I)$ and $\Sigma_{n}(J)$ are permutable. Thus, from the general theory of universal algebra ([3, $\$ 19, p .120])$, the map $a \mapsto\left([a] \Sigma_{n}(I),[a] \Sigma_{n}(J)\right)$ is an isomorphism of $L$ onto the direct product $L / \Sigma_{n}(I) \times L / \Sigma_{n}(J)$. Under this map $x^{*} \vee x^{* *}$ and 1 both correspond to $(1,1)$ and so $x^{*} \vee x^{* *}=1$. That is, $L \in B_{1}$ and so ( $x i$ ) implies (i).

COROLLARY 3.3. Let $J$ be a congruence-kermel in a stone lattice $L$. Then, $x \equiv y(R(J)), \quad(x, y \in L)$, if and only if $(x \vee a)^{* *}=(y \vee a)^{* *}$ for some $a \in J$.

Proof. By the previous theorem, $R$ permutes with $\theta_{\text {Lat }}(J)=\theta(J)$ and so $R(J)=R \circ \Theta_{\text {Lat }}(J) \quad($ Theorem $1.6(i v))$. Thus $x \equiv y(R(J))$ implies that $x^{* *}=z^{* *}$ and $z \vee a=y \vee a$ for some $a \in J$. In any Stone lattice, $(s \vee t)^{* *}=s^{* *} \vee t^{* *}$ is an identity, and so we obtain $(x \vee a)^{* *}=(y \vee a)^{* *}$ for some $a \in J$. The converse follows from the same identity and Theorem 1.6 (iii).

\section{References}

[1] William H. Cornish, "Normal lattices", J. Austral. Math. Soc. 14 (1972), 200-215.

[2] William H. Cornish, "Annulets and $\alpha$-ideals in a distributive lattice", J. Austral. Math. Soc. (to appear).

[3] George Grätzer, Universal algebra (Van Nostrand, Princeton, New Jersey; Toronto; London; Melbourne; 1968).

[4] George Grätzer, Lattice theory; first concepts and distributive Zattices (Freeman, San Francisco, 1971). 
[5] G. Grätzer and E.T. Schmidt, "Standard ideals in lattices", Acta Math. Acad. Sci. Hungar. 12 (1961), 17-86.

[6] Iqbal unnisa, "Permutable congruences in a lattice", Illinois J. Math. $10(1966), 235-239$.

[7] Joseph Kist, "Minimal prime ideals in commutative semigroups", Proc. London Math. Soc. (3) 13 (1963), 31-50.

[8] K.B. Lee, "Equational classes of distributive pseudo-complemented lattices", Conad. J. Math. 22 (1970), 881-891.

[9] T.P. Speed, "Some remarks on a class of distributive lattices", $J$. Austral. Math. Soc. 9 (1969), 289-296.

[10] T.P. Speed, "Two congruences on distributive lattices", BuZZ. Soc. Roy. Sci. Liège 38 (1969), 86-95.

School of Mathematical Sciences,

Flinders University of South Australia,

Bedford Park,

South Australia. 